\title{
A Novel Method for Planning and Visualization of Ablation Lines for Atrial Fibrillation Treatment
}

\author{
M Hastenteufel, I Wolf, C Christoph, S Yang, \\ T Boettger, M Vetter, HP Meinzer
}

\begin{abstract}
Deutsches Krebsforschungszentrum, Division Medical and Biological Informatics, Heidelberg, Germany
\end{abstract}

\begin{abstract}
The most common arrhythmia is atrial fibrillation. Beside surgery, intracardiac catheter ablation is used commonly for curative treatment. Navigation systems have been developed to reduce fluoroscopic time and to provide a more accurate positioning. Within these systems, standard 3D-views are used to show intracardiac catheters and ablated areas. We present a novel method to display spatial relations of catheter, anatomical landmarks and ablation lines in a mapped 2D-view. Furthermore, a planning of desired ablation lines is feasible. The method can be a valuable tool to support navigated ablation procedures for atrial fibrillation treatment.
\end{abstract}

\section{Introduction}

Atrial fibrillation (AF) is the most common arrhythmia, approximately 2.2 million people in USA are affect by it. Atrial fibrillation results in an increased risk of ischemic stroke due to thrombus formation in the left atrium [1]. It is guessed that $15 \%$ of all ischemic strokes are due to AF. There exist several therapy options, i.e. medicamentation, defibrillation, surgery and ablation [2].

The best possible curative treatment for atrial fibrillation is still subject of current research [3]. Highest curative rates (up to 97\%) are achieved by surgery (MAZE technique). Several incisions are made to the right and left atrium to effect necrotic tissue. The aim is to partition the myocardial tissue in small parts that are too small to maintain electrical re-entry waves. Newer studies showed that incisions can be limited to the left atrium. For intermittent AF, isolation of pulmonary veins can be sufficient in $90 \%$ [4, 5]. Surgery is highly invasive, sternotomy and cardiopulmonary bypass are used. Some attempts were made to apply these techniques minimally invasive [6].

Ablation techniques using intracardiac catheters were developed to overcome the highly invasive nature of surgical techniques. Small catheters are used to create lesions by applying different sources of energy, e.g. radiofrequenzy, cryo, laser or ultrasound. Curative rates of catheter ablations are not as high as for surgery, but those interventions are less invasive than surgery. However, catheter ablation takes a long time (up to five hours) and results in high $\mathrm{x}$-ray exposure due to fluoroscopic guidance (fluoroscopic time is up to one hour and more) [7].

To reduce flouroscopy time and to enhance catheter guidance, several electroanatomical intracardiac navigation systems have been developed [8], i.e. CARTO (Biosense Webster, USA), LocaLisa (Medtronic, USA) or Ensite NavX (Endocardial Solutions, USA). The application of such navigation systems results in reduced $\mathrm{X}$-ray exposure. Beside visualizing relative catheter positions, some systems allow to create a virtual model of the heart and to visualize catheter positions in relation to this model.

Current research aims at the integration of patient individual anatomy, obtained by preoperative magnetic resonance imaging (MRI) or computed tomography imaging (CT), into these systems [9, 10]. Knowledge of the patient's individual anatomy is important to avoid complication such as pulmonary vein stenosis [11], which occurs when ablating to close to pulmonary vein orifices.

The intracardiac navigation systems that are currently available visualize catheter positions in 3D views [10]. However, orientation in $3 \mathrm{D}$ views is difficult due to the missing 3rd dimension of the screen. In other medical fields like image guided dental implantology, visualization methods exist that project $3 \mathrm{D}$ scenes onto $2 \mathrm{D}$ views.

In this paper we present a novel 2D visualization method to project the left atrium, anatomical landmarks, intracardiac catheters as well as made ablation lines onto a 2D anatomical map. Before intervention, desired ablation lines can be planned in this map and are visualized in a conventional $3 \mathrm{D}$ view. Using this $2 \mathrm{D}$ anatomical map results in a more precise positioning of the ablation device and enables an ablation planning with target/actual comparison of catheter positions. 


\section{Material and methods}

\subsection{Imaging}

Prerequisite is a preoperative or intraoperative imaging of the required cardiac structure. Preoperative imaging can be performed using MRI or CT. Figure 1 shows a preoperative CT image (left) and the segmented left atrium with the pulmonary veins and the left atrial appendage. The segmentation for that visualization has been done manually with a custom-made segmentation software developed at our department[12].
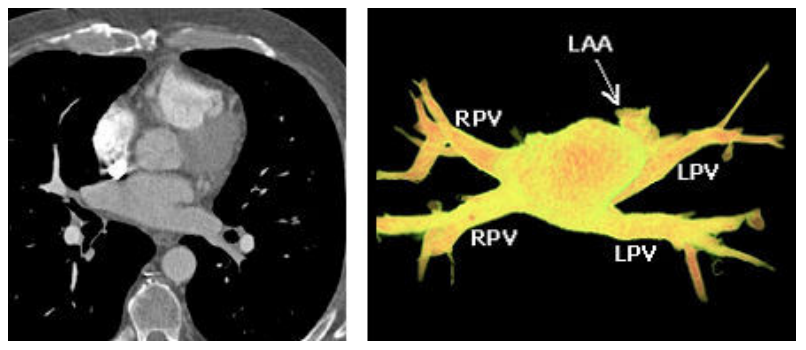

Figure 1. Left: cardiac CT. Right: segmented left atrium with pulmonary veins (RPV, LPV) and left atrial appendage (LAA).

Intraoperative imaging can be performed for example by means of 3D transesophageal echocardiography. Transesophageal echocardiography has become more important as minimally invasive techniques have been added to cardiac surgery [13].

\subsection{Thin-plate spline approximation}

The anatomy of the left atrium and the pulmonary veins is rarely described in textbooks. Recent studies deal with this topic [14]. However, the left atrium is predominantly convex and can be approximately modeled as an elliptical structure. Therefore, an ellipsoid-like model is fitted to the left atrium to model its anatomy. Anatomical landmarks such as

- pulmonary veins

- left atrial appendage

- mitral valve annulus

- arbitrary endocardial points

are interactively defined. The center of gravity $c$ of these points is calculated and used as the center of an initial sphere with radius $r \leq \min _{i}\left(\left\|c, t_{i}\right\|\right)$, where $t_{i}$ are the given landmarks. Now, the sphere is fitted through the anatomical landmarks using a thin-plate spline transformation (TPST) [15]. For that, the anatomical landmarks $t_{i}$ are projected onto the sphere to obtain a set of source landmarks $s_{i}$ needed by the TPST, see figure 2 .
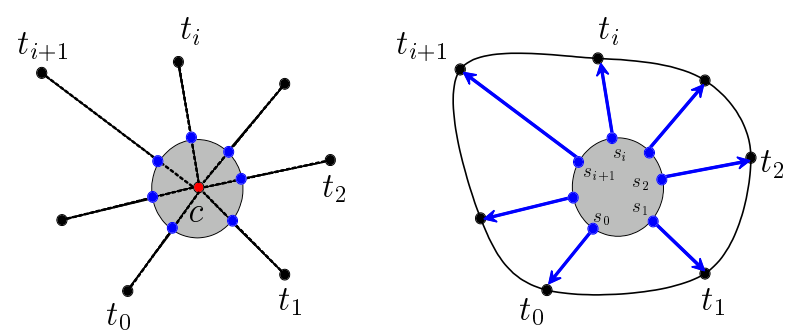

Figure 2. Fitting an initial sphere to anatomical landmarks $t_{i}$ by means of a thin-plate spline transformation. The landmarks are projected onto the sphere for estimating source landmarks $s_{i}$.

\subsection{Mapping}

Now, the surface of the ellipsoid-like model has to be mapped to a 2D plane. This is similar to the well known mercador projection to display the globe in a $2 \mathrm{D}$ world map. Figure 3 illustrates the mapping procedure.

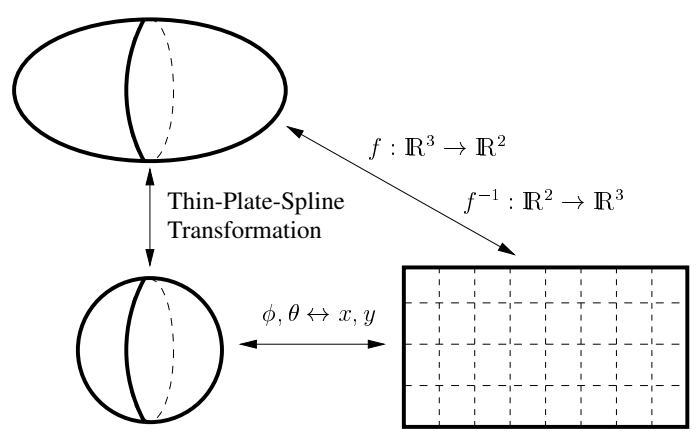

Figure 3. Mapping 3D coordinates of the ellipsoid model to $2 \mathrm{D}$ coordinates of the $2 \mathrm{D}$ anatomical map $(\phi, \theta$ are cylindrical coordinates, $x, y$ cartesian coordinates).

The resulting map is isogonal but length preservation is not assured. However, this is not obstructive to reveal the relation of desired ablation lines, already made ablation lines and anatomical landmarks as well as to verify the continuity of ablation lines.

\subsection{Planning}

There are several ablation patterns concerning atrial fibrillation treatment described in literature [16]. Some authors propose to ablate around all four pulmonary veins, some to ablate around the left and right pulmonary veins and to connect these lines. Other authors propose to ablate around each pulmonary vein themselves. Additionally, a circle around the left atrial appendage as well as a 
connection of the pulmonary vein area to the mitral valve annulus is usually ablated. Figure 4 shows a typical ablation pattern.

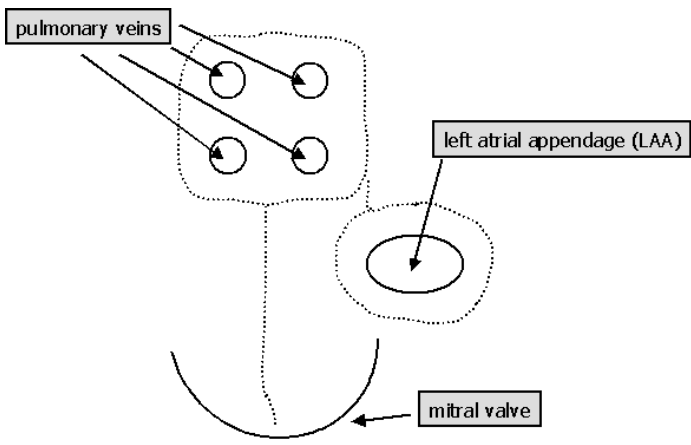

Figure 4. Typical ablation pattern used for the treatment of atrial fibrillation.

It is important to ablate not too close to the pulmonary vein orifice due to the risk to cause pulmonary vein stenosis [11]. Taken the patient's anatomy into consideration, an individual planning of ablation lines can be beneficial to avoid that.

Using our tool, desired ablation lines can be drawn in the $2 \mathrm{D}$ anatomical map and are visualized simultaneously in the $3 \mathrm{D}$ view. If the intracardiac ablation device is registered to the image, actual ablations can be visualized and verified with respect to the desired ablation lines.

\subsection{Implementation}

The described methods are implemented using our framework for medical imaging applications (MITK, [17]). It uses the well known toolkits Qt for the graphical user interface (Trolltech, Norway), VTK for visualization (Kitware, USA) and ITK for segmentation and registration (Kitware, USA). The mapping procedure has been implemented using transformations provided by VTK (vtkThinPlateSplineTransform, vtkSphericalTransform).

Due to the implementation within our medical imaging framework MITK, an interactive usage became feasible. Interactions in the 2D anatomical map, e.g planning of desired ablation lines, immediately affect the 3D view.

\section{Results}

\subsection{Thin-plate spline approximation}

Figure 5 shows the approximation of the left atrium by means of a thin-plate spline transformation. For better illustration, the approximation is shown in relation to a manually segmented atrium. On the right hand side of figure 5 the initial sphere is shown in relation to the approximated atrium. It has been turned out that the natural anatomical landmarks (pulmonary veins, left atrial appendage) are insufficient. In figure 5 approximately 25 additional endocardial landmarks were used. Figure 6 shows the approximation overlaid to the original CT slices.
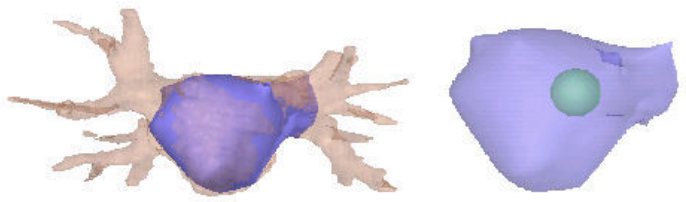

Figure 5. Approximated thin-plate spline model in relation to manually segmented left atrium (left) and initial sphere in relation to thin-plate spline model (right).

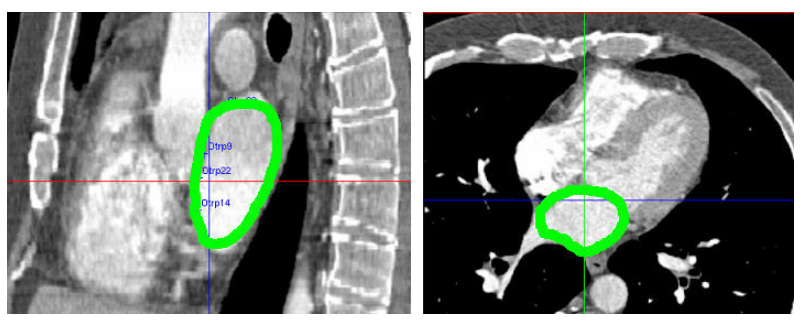

Figure 6. With TPST segmented left atrium overlaid to original slices (manually enhanced for better visualization).

\subsection{Mapping and planning}

Figure 7 shows an example of the planning functionality. An ablation pattern has been interactively drawn around two pulmonary veins in the $2 \mathrm{D}$ anatomical map. This ablation pattern is transformed and visualized in the $3 \mathrm{D}$ view. Figure 8 shows the mapped visualization of made ablation lines. The spatial relation, especially the continuity of multiple ablation lines can be better estimated in the $2 \mathrm{D}$ anatomical map than in the $3 \mathrm{D}$ view.

\subsection{Application}

The method has been integrated into a currently developed navigation system for a new kind of ablation devices for minimally invasive surgical atrial fibrillation treatment. Imaging is performed by means of intraoperative 3D ultrasound. The navigation system as well as the new $2 \mathrm{D}$ visualization and planning tools are currently evaluated in in-vitro trials under realistic physiological conditions. 

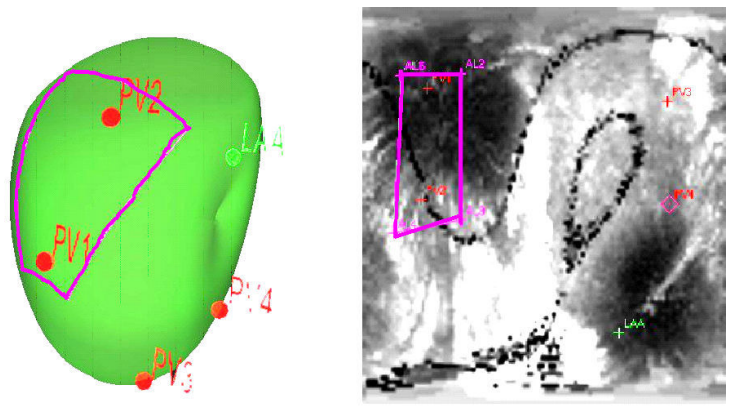

Figure 7. Planning of ablation lines: Desired ablation lines can be drawn in the 2D anatomical map and are visualized in the $3 \mathrm{D}$ view.

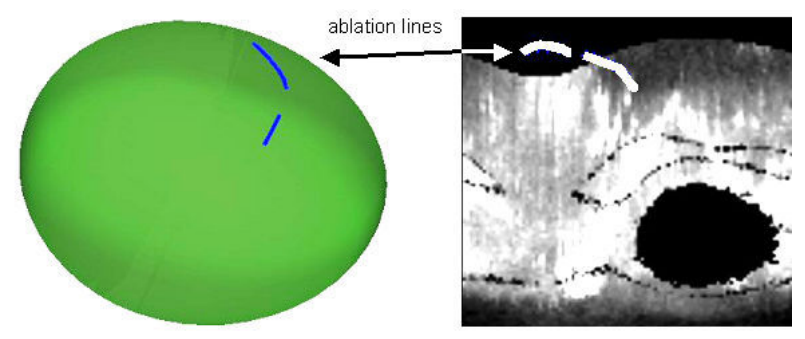

Figure 8. Visualization of made ablation lines in the 2D anatomical view.

\section{Conclusion}

In this paper, a novel method for visualization and planning of ablation lines has been presented. Due to potential complication when ablating too close to pulmonary veins and absolute essential continuous ablation lines, our new method can be a valuable tool for catheter ablation or minimally invasive surgical ablation for atrial fibrillation treatment. The value of the tool is currently investigated in in-vitro trials using a navigation software developed at our institution to guide minimally invasive surgical devices for atrial fibrillation treatment.

\section{Acknowledgement}

The department of cardiac surgery of the university hospital Heidelberg, Germany, provided the cardiac images.

\section{References}

[1] Ferro J. Cardioembolic stroke: An update. Neurology 2003; 2:177-188.

[2] Falk R. Atrial fibrillation. N Engl J Med 2001; 344(14):1067-1078.

[3] Gaita F, Riccardi R. Lone atrial fibrillation: Transcatheter or minimally invasive surgical approaches? J Am Coll Cardio 2002;40(3):481-483.
[4] Haissaguerre M, Jais P, Shah D, Takahashi A, et al. Spontaneous initiation of atrial fibrillation by ectopic beats originating in the pulmonary veins. N Engl J Med 1998; 339(10):659-66.

[5] Cox J. Atrial fibrillation I: A new classification scheme. H Thorc Cardiovasc Surg 2003;126:1686-92.

[6] Kottkamp H, Hindricks G, Autschbach R, Krauss B, et al. Specific linear left atrial lesions in atrial fibrillation: Intraoperative radiofrequency ablation using minimally invasive surgical techniques. J Am Coll Cardio 2002; 40(3):475-480.

[7] Macle L, Weerasooriya R, Jais P, Scavee C, et al. Radiation exposure during radiofrequency catheter ablation for atrial fibrillation. PACE 2003;26:288-291.

[8] Ben-Haim S. Catheter navigation in modern electrophysiology. J Cardiovasc Electrophysiol 2000; 11(1191-1195).

[9] Dickfeld T, Calkins H, Zviman M, Kato R, et al. Anatomic stereotactic catheter ablation on three-dimensional magnetic resonance images in reat time. Circulation 2003; 108:2407-2413.

[10] Morady F. Catheter ablation of supraventricular arrhythmias: State of the art. PACE 2004;27:125-142.

[11] Arentz T, Jander N, von Rosenthal J, Blum T, et al. Incidence of pulmonary vein stenosis 2 years after radiofrequency catheter ablation of refractory atrial fibrillation. European Heart Journal 2003;24:963-969.

[12] Kunert T, Heimann T, Schröter A, Schöbinger M, et al. An interactive system for volume segmentation in computerassisted surgery. In Proc. SPIE Medical Imaging, volume 5367. 2004; In Press.

[13] Lehot J, Blanc P, Arvieux C, Jegaden O. Transesophageal echocardiography for minimally invasive cardiac surgery. J Cardiovasc Surg 2001;42:291-5.

[14] Kato R, Lickfett L, Meininger G, Dickfeld T, et al. Pulmonary vein anatomy in patients undergiong catheter ablation in atrial fibrillation: Lessons learned by use of magnetic resonance imaging. Circulation 2003;107:20042010.

[15] Bookstein F. Principal warps: Thin-plate splines and the decomposition of deformations. IEEE Trans Pattern Anal 1989;11(6):567-585.

[16] Cox J. Cardiac surgery for arrhthmias. PACE 2004;27:266282.

[17] Wolf I, Vetter M, Wegner I, Boettger T, Nolden M, Schoebinger M, Hastenteufel M, Kunert T, Meinzer H. The medical imaging interaction toolkit (mitk). Medical Image Analysis 2004; In press.

Address for correspondence:

Mark Hastenteufel

Deutsches Krebsforschungszentrum

Im Neuenheimer Feld 280

69120 Heidelberg, Germany

M.Hastenteufel@dkfz.de 